\title{
Phenomenal Simulation Modelling of X-Ray Diffraction Patterns of Pyrite Ash Bioprocessed in Acetobacter aceti Contained Media
}

Bolu Abant İzzet Baysal University, Department of Chemical Engineering, Bolu, Turkey

\begin{abstract}
Phenomenal profiling functions were applied for the simulation of an X-ray diffraction (XRD) pattern of pyrite ash bioprocessed in Acetobacter aceti contained growth media. The full width at half maximum (FWHM) values were estimated for each pattern of pyrite ash, and the Bragg equation was used to determine the atomic layer spacing, as well crystallite size and strain broadening were accomplished by applications of Scherrer and Williamson-Hall equations. Errors in line broadening were correlated with the FWHM equations of the generalised profiling functions, and the microstrain $(\varepsilon)$ was estimated with the relation between the integral breadth $\left(\beta_{\mathrm{i}}\right)$ and $\cos \theta$. Furthermore, the XRD pattern of a discrete peak was 3D simulated using Gaussian, Lorentzian, Pearson VII, pseudo-Voigt, and Voigt functions. The Gaussian function with a round top peak was designated the most suitable profile simulation by offering the maximum peak height, $I_{\max }$ and tails comparable to the experimental peak.
\end{abstract}

\section{Keywords}

X-ray diffraction pattern, crystallite size and strain broadening, FWHM investigation, profiling modelling, pattern simulation, pyrite ash, Acetobacter aceti

\section{Introduction}

Phenomenal simulation functions were applied to X-ray diffraction (XRD) patterns to better understand the empirical outcomes of crystal structures and simulate the peak profiles. The essentials of diffractometer system techniques are based on the formation of incident X-rays at several wavelengths; the rays interact with the electrons of the atoms in the crystal, and the diffracted beams pattern of $X$-rays from the crystal lattice at relevant angles then produce peaks of diffraction intensity. The peak profile is shaped relatively with the angle of the diffracted rays enduring essential structures. The total peak area under the curve indicates peak intensity and specifies the full amount of complete diffracted X-ray photons. ${ }^{1,2,3,4}$

X-ray diffraction is practically applied for composition determination of mineral samples such as pyrite ash, which was used in this study as the mineral media for growth of Acetobacter aceti, which lowers the $\mathrm{pH}$ of the media and consumes sulphur and other minerals of the ash.

\subsection{Profiling functions}

The X-ray waves are diffracted by electrons of the atoms, and the wavelength and angle of the diffracted $X$-ray beams are defined as the intrinsic parameters used to describe the shape profiling of a diffraction peak. The wavelength of the beam is related to the atomic layer spacing and reflection angle of the beam, which is a central geometric phenomenon defined by Bragg as the wavelength equation. The equation of Bragg's Law verifies the atomic structure of crystals and describes the reflection of the X-ray beams by the crystals at certain angles of frequency.

The Bragg wavelength equation is formulised as follows:

$$
\lambda=2 d \sin \theta
$$

where $\lambda$ is wavelength, $d$ represents spacings, and $\theta$ is the Bragg angle.

The wavelength equation is used to determine the relationship between the wavelengths, the atomic layer spacing, and the diffraction angle of the diffracted X-rays. The $d$-spacing of each peak for crystallite mineral can be obtained by the rate of the wavelength over the Bragg angle.

$X$-ray diffraction peaks determined at various reflection angles, which are related to the size of crystallite particles. ${ }^{2}$ The beams of the diffracted X-rays occur depending on the specific reflection angles, which occur by the atomic layer spacing characteristics of the crystals. The broadening of each diffraction peak was measured and plotted as a function of the Bragg angle for the determination of the strain and particle size of a nanoscale $\mathrm{Al}_{2} \mathrm{O}_{3} / \mathrm{ZrO}_{2}$ composite. ${ }^{5}$

The broadening of XRD peaks arises from the size of the diffraction domain. The width of XRD peaks under a specific reflection angle is related to the size of the crystallite particle by the Scherrer equation phenomenon, which is used for crystallite size broadening and for the determination of grain size from broadened peaks.

$$
L=\frac{K \lambda}{\beta(2 \theta) \cos \theta}
$$


where, $L$ indicates crystallite size, $K$ is constant, $\lambda$ is the wavelength, $\beta$ denotes line broadening, and $\theta$ is the Bragg angle.

The highest intensity point of the peak represents maximum height of the peak, which is symbolized as $I_{\max }$ and the peak width is a measure of the broadness of the peak that at large angles, such as $0.2^{\circ}$ angle steps, designate a broad peak. The width of the peak is a measure of the broadness of the peak, where a horizontal line at the half maximum peak height $\left(1 / 2 I_{\max }\right)$ is denoted as the full width at half maximum (FWHM). The integral breadth $\left(\beta_{\mathrm{i}}\right)$ is a function of the FWHM which is a suitable equation for the peak profiling.

The integral breadth $\left(\beta_{\mathrm{i}}\right)$ peak profiling parameter as a function of FWHM is defined as follows:

$$
\beta_{\mathrm{i}}=\frac{\pi(\mathrm{FWHM})}{2}
$$

Any deformation in mineral structure causes strain broadening, though the microstrain $(\varepsilon)$ is proportional to the integral breadth and the Bragg angle:

$$
\varepsilon=\frac{\beta_{\mathrm{i}} \cos \theta}{4}
$$

This article proposes a new theory of X-ray scattering that has particular relevance to the powder diffraction. The underlying concept of this theory is that the scattering from a crystal or crystallite is distributed throughout the space: this leads to the effect that enhanced scatter can be observed at the 'Bragg position' even if the 'Bragg condition' is not satisfied. The scatter from a single crystal or crystallite, in any fixed orientation, has the fascinating property of contributing simultaneously to many 'Bragg positions'. It also explains why diffraction peaks are obtained from samples with very few crystallites, which cannot be explained with the conventional theory. The intensity ratios for a Si powder sample are predicted with greater accuracy and the temperature factors are more realistic. Another consequence is that this new theory predicts a reliability in the intensity measurements which agrees much more closely with experimental observations compared to the conventional theory that is based on 'Bragg-type' scatter. The role of dynamical effects (extinction etc.) is discussed and how they are suppressed with diffuse scattering. An alternative explanation for the Lorentz factor is presented that is more general and based on the capture volume in diffraction space. This theory, when applied to the scattering from powders, will evaluate the full scattering profile, including peak widths and the 'background'. The theory should provide an increased understanding of the reliability of powder diffraction measurements, and may also have wider implications for the analysis of powder diffraction data, by increasing the accuracy of intensities predicted from structural models.

The crystallite size broadening $\left(\beta_{c}\right)$ extracted from the Scherrer formula, and the strain broadening $\left(\beta_{\mathrm{s}}\right)$ are angle dependent and differ with the Bragg angle. ${ }^{1,4}$ The crystallite size and strain broadening are relative to $(1 / L \cos \theta$ and $(\tan \theta)$, respectively, as symbolised in the following functions:

$$
\begin{aligned}
& \beta_{\mathrm{c}}=\frac{K \lambda}{L \cos \theta} \\
& \beta_{\mathrm{s}}=C \varepsilon \tan \theta
\end{aligned}
$$

The sum of crystallite size broadening and strain broadening is stated as the Williamson and Hall convolution phenomenon, and the graph of the crystallite size vs strain broadening is known as the Williamson-Hall plot which provides strain component $(C \varepsilon)$ from the slope and the size component $(K \lambda / L)$ from the intercept. ${ }^{6,7}$

The linearised Williamson-Hall equation is given as follows:

$$
\beta_{\mathrm{tot}} \cos \theta=C \varepsilon \sin \theta+\frac{K \lambda}{\beta \cos \theta}
$$

\subsection{Simulation functions}

The simulation of XRD pattern can be effectively visualized using phenomenal profiling functions which are able to designate the peak height, peak shape, or line profile. The modelling functions of Gaussian and the Lorentzian are the basic simulation equations applied for fitting peaks of the experimental XRD analysis data. The functions are modified to obtain generalised profiling functions by making of power form, combination, and convolution for modelling of the specific diffraction peak shapes. Likewise, the derived Pearson VII, pseudo-Voigt, and Voigt functions are improved by the peak profile simulation parameters. ${ }^{4,8}$ The power form of Lorentzian function raised to $m$ yields the Pearson VII function, and the pseudo-Voigt function is a linear combination of the Gaussian and Lorentzian functions. The Voigt function is a convolution approach of the Gaussian and Lorentzian functions associated with the Faddeyeva complex error function. ${ }^{9-14}$

The occurrence of the errors in size and strain broadening is usually reported in terms of standard deviation $(\sigma)$ correlated FWHM equations of the generalised profiling functions.

The generalised profiling functions, and their related FWHM functions are listed in Table 1.

The tails of the peak between the angles characterise the limits at the left and right side of the peak, and as it asymptotically approaches zero intensity, they can provide a sensitivity to fit the peak using a mathematical simulation function. The peak asymmetry is defined as either symmetrical or asymmetrical depending on the identical reflection of the lines at either side.

$X$-ray diffraction and other electron microscopy techniques were used in an extensive study to determine the chemical composition and nanomineralogical assemblage of pyrite ash samples, along with the submicron, ultrafine, and nanoparticle characterization and stoichiometric composition of a number of major and trace elements and molecules. ${ }^{15}$ The mineralogical reduction and the $\mathrm{Fe}_{3} \mathrm{O}_{4}$ transformations 
Table 1 - Simulation and FWHM functions

\begin{tabular}{c|c|c}
\hline Models & Simulation functions & FWHM functions \\
\hline Gauss & $G(x)=\mathrm{e}^{\left(-\mathrm{x}^{2}\right)}$ & $\sqrt{2 \ln 2} \sigma$ \\
\hline Lorentz & $L(x)=\frac{1}{\left(x^{2}+1\right)}$ & $2 \sigma$ \\
\hline Pearson VII & $P(x)=\frac{1}{\left(x^{2}+1\right)^{m}}$ & $2 \sigma \sqrt{2^{1 / m}-1}$ \\
\hline pseudo-Voigt & $p V(x)=\mathrm{e}^{\left(-\mathrm{x}^{2}\right)}+\frac{1}{\left(x^{2}+1\right)}$ & $2 \sigma$ \\
\hline Voigt & $V(z)=\mathrm{e}^{\left(z^{2}\right)} \operatorname{erfc}(z)$ & $3.6 \sigma$ \\
\hline
\end{tabular}

in pyrite ash samples discarded from the roasting of pyrite ores, processed during production at a sulphuric acid plant, were investigated by XRD analysis and the quantity of $\mathrm{Fe}_{3} \mathrm{O}_{4}$ was determined as $61-73 \%$ in the pyrite ash samples. ${ }^{16}$

A. aceti has the capability of living in mineral media and generating proton flux, which decreases the $\mathrm{pH}$ of the liquid medium. A. aceti was used for its proton $\left(\mathrm{H}^{+}\right)$motive force for acetic acid production. ${ }^{17}$ The $\mathrm{pH}$ effect on the rates of iron and sulphur oxidation by bioleaching organisms was accomplished, and the pyrite ash samples were used for the successful incubation of Saccharomyces cerevisiae and Acetobacter aceti, at $\mathrm{pH}$ ranges of 3-6, without the occurrence of toxicities. ${ }^{18-20}$

\subsection{Experimental details}

The calcinated pyrite mineral samples were bioprocessed in A. aceti-comprising media at $37^{\circ} \mathrm{C}$ and $\mathrm{pH} 3.6$ for 20 days in a batch-type liquid-state bioprocess. The ash samples with high iron content ( $w=61 \%$ ) were provided from the Eti Mining Bandırma Borax Plant in Turkey and A. aceti strains of commercial vinegar were used as the incubation microorganisms. The particle size of pyrite ash was determined using the Malvern Instruments Mastersizer 2000 analyser. The incubation medium contained $5 \%$ pyrite ash, yeast-extract agar, and ethanol. The XRD pattern data of the pyrite ash samples were collected using Rigaku diffractometer with $\mathrm{Cu}-\mathrm{K} \alpha$ radiation $(\lambda=1.54056 \AA)$. The pyrite mineral ash samples were investigated by instrumental XRD profile analysis with the diffraction peaks between $20^{\circ}$ and $90^{\circ} 2 \theta$ angles. The essentials of MATLAB tools were used for calculations and simulation of XRD pattern.

\section{Results}

The growth of $A$. aceti strains in 10-100 $\mu \mathrm{m}$ particle size pyrite mineral ash containing liquid state media was effectually observed during incubation. The initial $\mathrm{pH}$ of the growth medium declined from 6.7 to 3.4 , thereby stim- ulating the redox potential and biochemical oxidation, which activated changes in mineral compositions.

The instrumental XRD profile analysis of the structural composition of the pyrite mineral ash samples was successfully accomplished, as shown in Fig. 1, with the diffraction peaks between $20^{\circ}$ and $80^{\circ} 2 \theta$ angles.

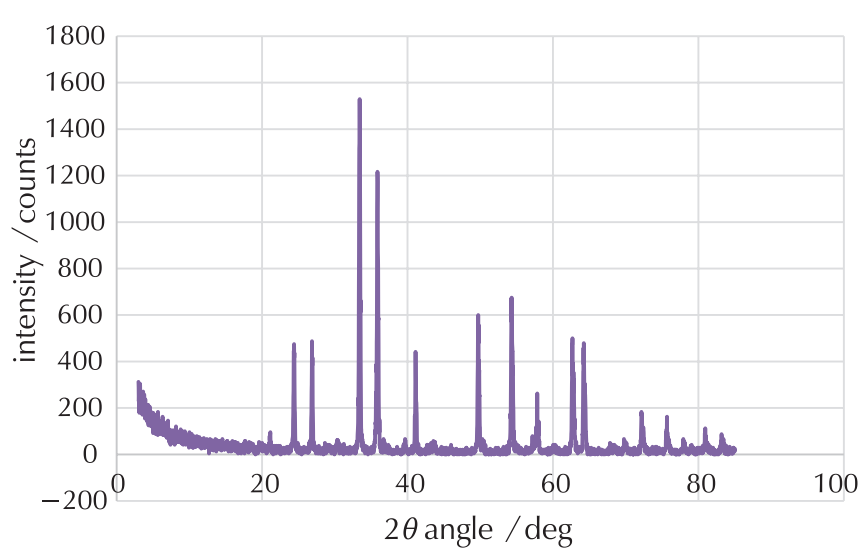

Fig. 1 - X-ray diffraction pattern of pyrite ash at discrete diffraction angles

\subsection{Simulation of the diffraction profiles}

Size of the peak at $0.2^{\circ}$ per step indicated a broad peak, and the peak tailed between $\left(33.12^{\circ}\right.$ and $\left.33.66^{\circ}\right) 2 \theta$ angles, as characterised in Fig. 2, which was chosen for the simulation in this study.

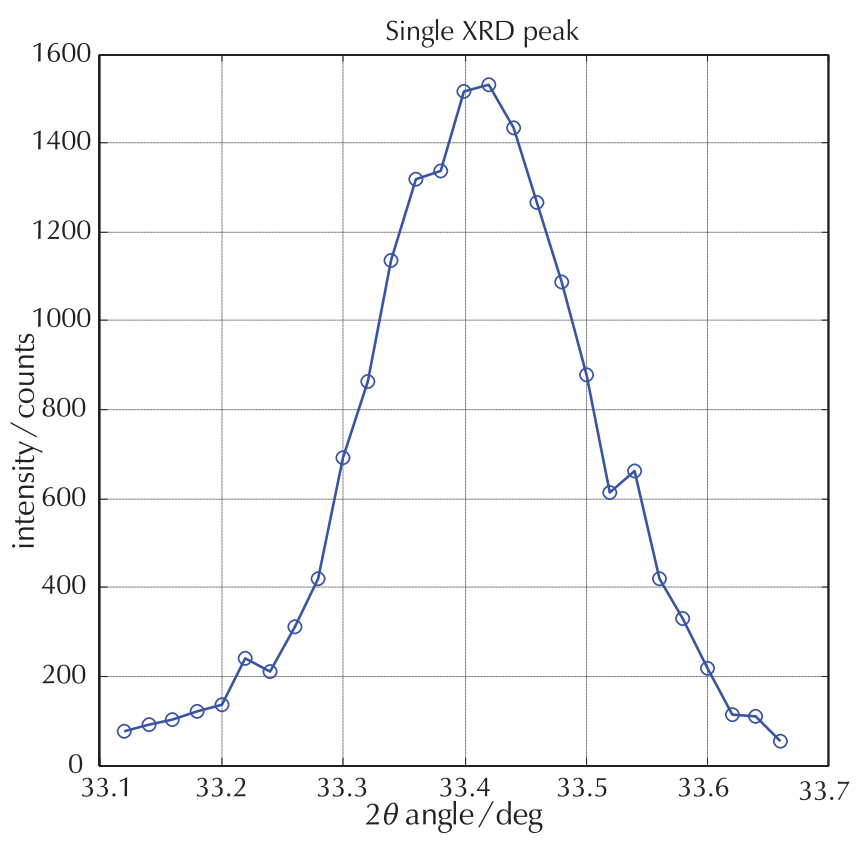

Fig. 2 - Single XRD peak profile of pyrite ash 
The highest intensity value -maximum height of the peakwas determined as $I_{\max }=1529.17$, and the half maximum peak was found to be $1 / 2 I_{\max }=764.58$. The FWHM was observed as between $\left(33.3^{\circ}-33.54^{\circ}\right) 2 \theta$ angles at $1 / 2 I_{\max }$.

Fig. 3 represents the error estimation graph of standard deviation $(\sigma)$ associated FWHM estimations for each generalised profiling function as FWHM functions of Gaussian (FWHMG), Lorentzian (FWHML), Pearson VII (FWHMP), pseudo-Voigt (FWHMPV), and Voigt (FWHMV) vs theta angle.

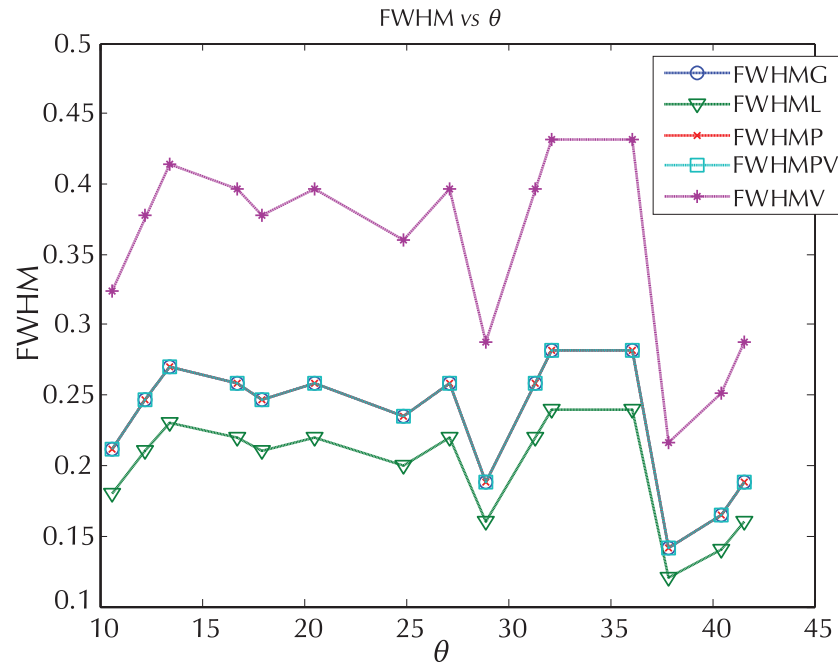

Fig. 3 - Error estimation graph of standard deviation $(\sigma)$ associated FWHM estimations for each generalised profiling function vs theta angle

The relation between microstrain $(\varepsilon)$, crystallite size $(L)$, and Bragg angle $(\theta)$, as well as FMHM, crystallite size $(L)$ and Bragg angle $(\theta)$ are presented in Figs. $4 \mathrm{a}$ and $4 \mathrm{~b}$.

The phenomenal Williamson-Hall approach was applied to conceptualise a plot of $\left(\beta t_{\mathrm{ot}} \cos \theta\right)$ vs $\sin \theta$ using the calculated data, as shown in Fig. 5. The parameters of $C \varepsilon$ and the $K \lambda / L$ values were assessed from the generated Williamson-Hall graph. In addition, the size of crystallite was approximated using the Scherrer equation by taking the constant $\mathrm{K}$ averaged 0.94 for spherical crystals with cubic symmetry.

The estimated values of $C \varepsilon$ and $K \lambda / L$ were used for the determination of $\beta_{\text {tot }}$ which was applied to generate a graph of $\beta_{\text {tot }}$ FWHM vs Bragg angle $(\theta)$, as depicted in Fig. 6.

Fig. 7 illustrates the microstrain $\left(\varepsilon\left(\beta_{i}, \cos \theta\right)\right)$, which was estimated by a straight relation between the integral breadth $\left(\beta_{\mathrm{i}}\right)$ and $\cos \theta$.

The relationships between the standard deviation $(\sigma)$ related FWHM, $I_{\max }$ vs d-spacing estimations are presented in sub-graphs in Figs. 8 and 9. The FWHM approximations of
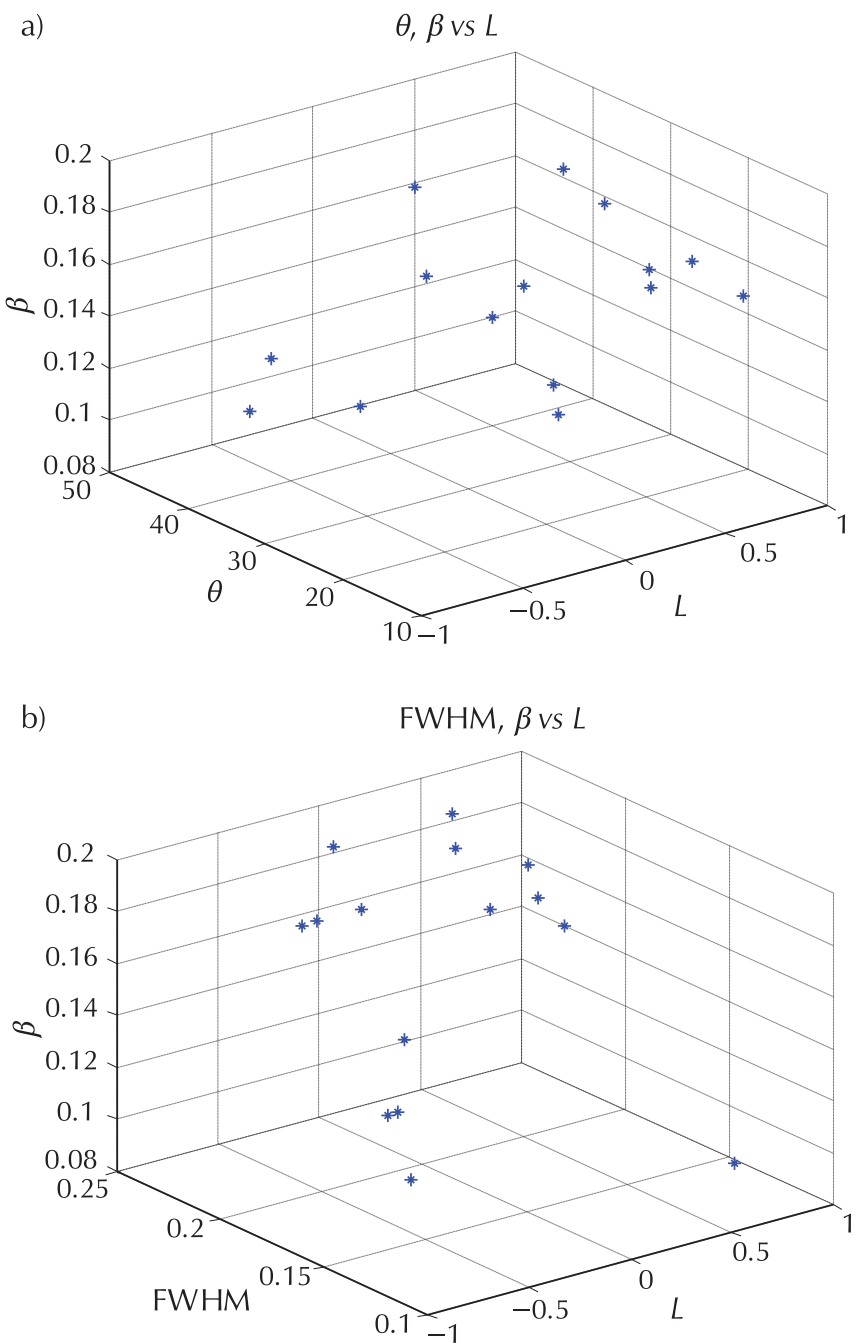

Fig. 4 - a) Graph of Bragg angle $(\theta)$, line broadening $(\beta)$, crystallite size $(L)$, and b) graph of FMHM, Bragg angle $(\theta)$ and crystallite size $(L)$

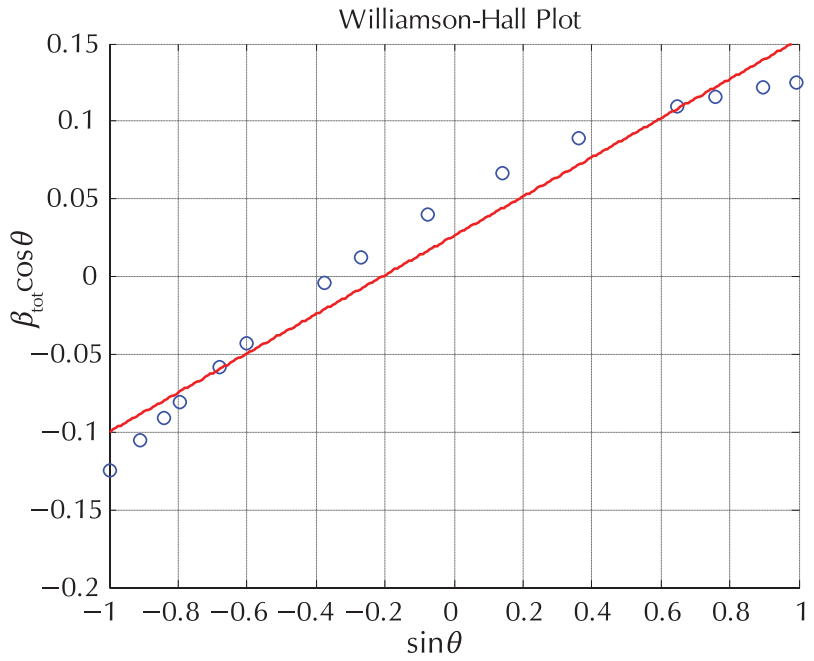

Fig. 5 - Williamson-Hall plot of $\left(\beta_{\mathrm{tot}} \operatorname{Cos} \theta\right)$ vs $\sin \theta$ 
Gaussian, Lorentzian, pseudo-Voigt, and Voigt functions, $I_{\max }$ vs $d$-spacing are presented in Fig. 8, and Fig. 9 represents a graph of the Pearson VII FWHM, $I_{\max }$ vs $d$-spacing for powers of $m=1,3,6,9$.

The individual peak of the XRD pattern of pyrite ash bioprocessed in $A$. aceti contained growth media was 3D simulated, as illustrated in Figs. 10 and 11, applying funda- mental approximation functions such as Gaussian, Lorentzian, Pearson VII, pseudo-Voigt, and Voigt equations.

Fig. 10 displays symmetrical round-top Gaussian, and sharp-top Lorentzian, pseudo-Voigt, and Voigt simulation of the XRD pattern.

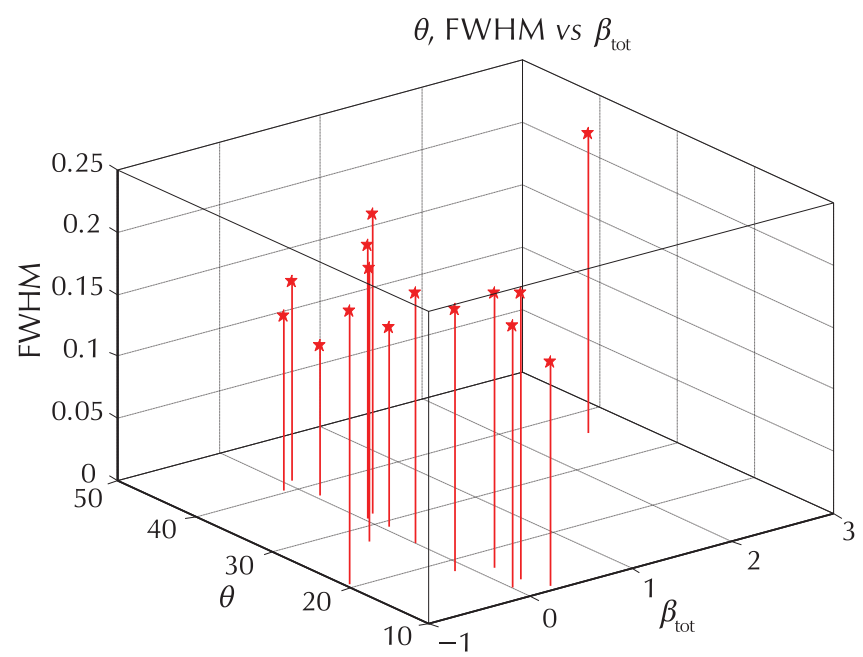

Fig. 6 - Plot of Bragg angle ( $\theta)$, FWHM vs $\beta_{\text {tot }}$
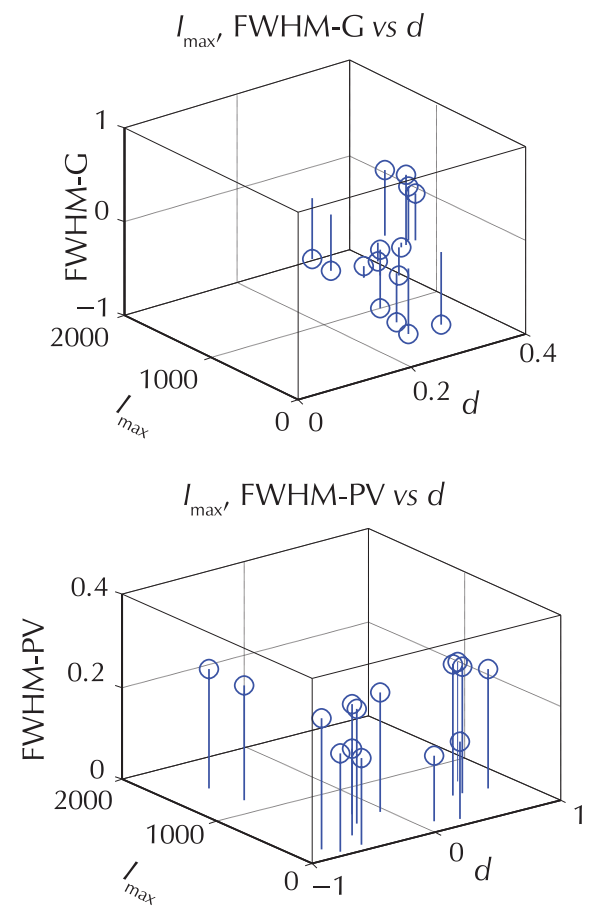

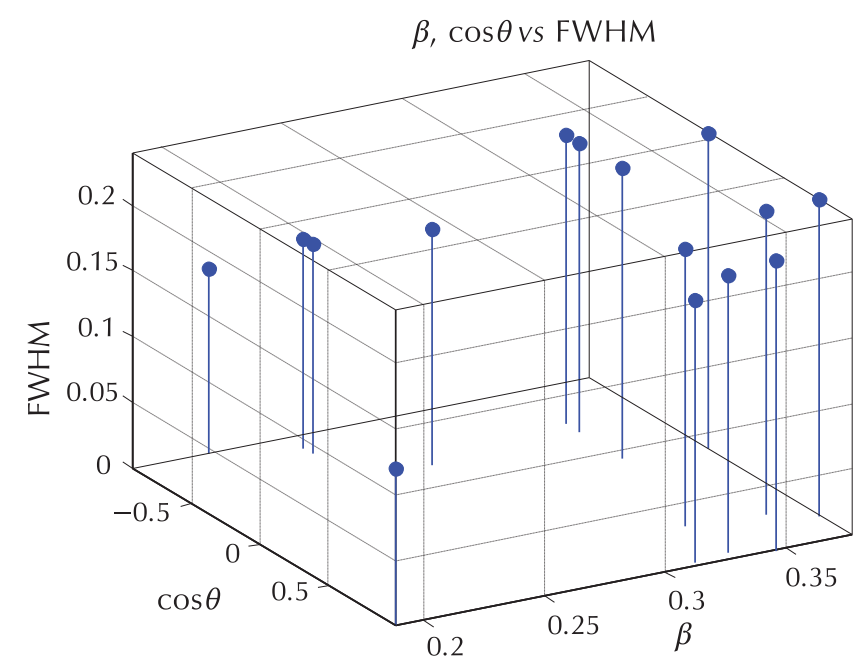

Fig. 7 - Graph of microstrain ( $\varepsilon$ vs FWHM
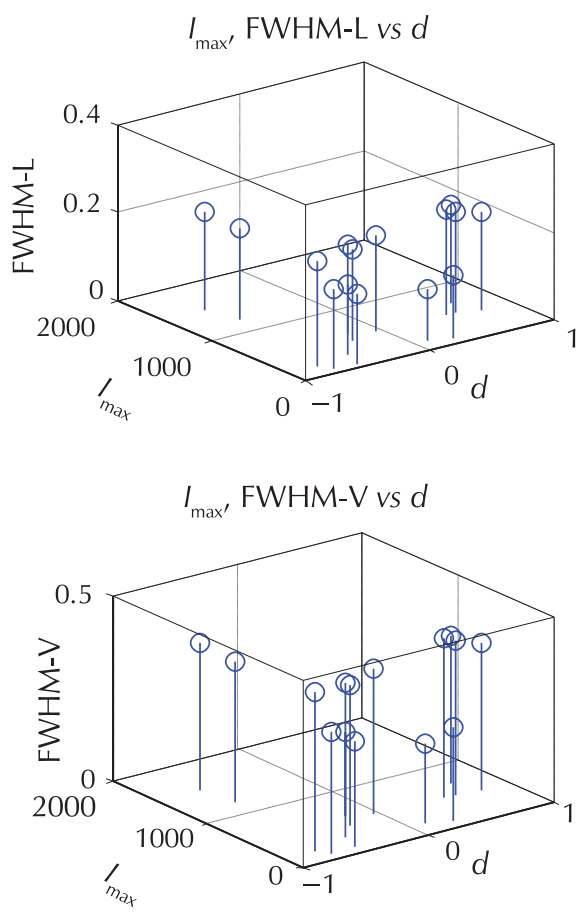

Fig. 8 - FWHM visualisations of Gaussian (FWHMG), Lorentzian (FWHML), Pearson VII (FWHMP), pseudo-Voigt (FWHMPV), and Voigt (FWHMV) functions FWHM, I $I_{\max }$ vs $d$-spacing plot 

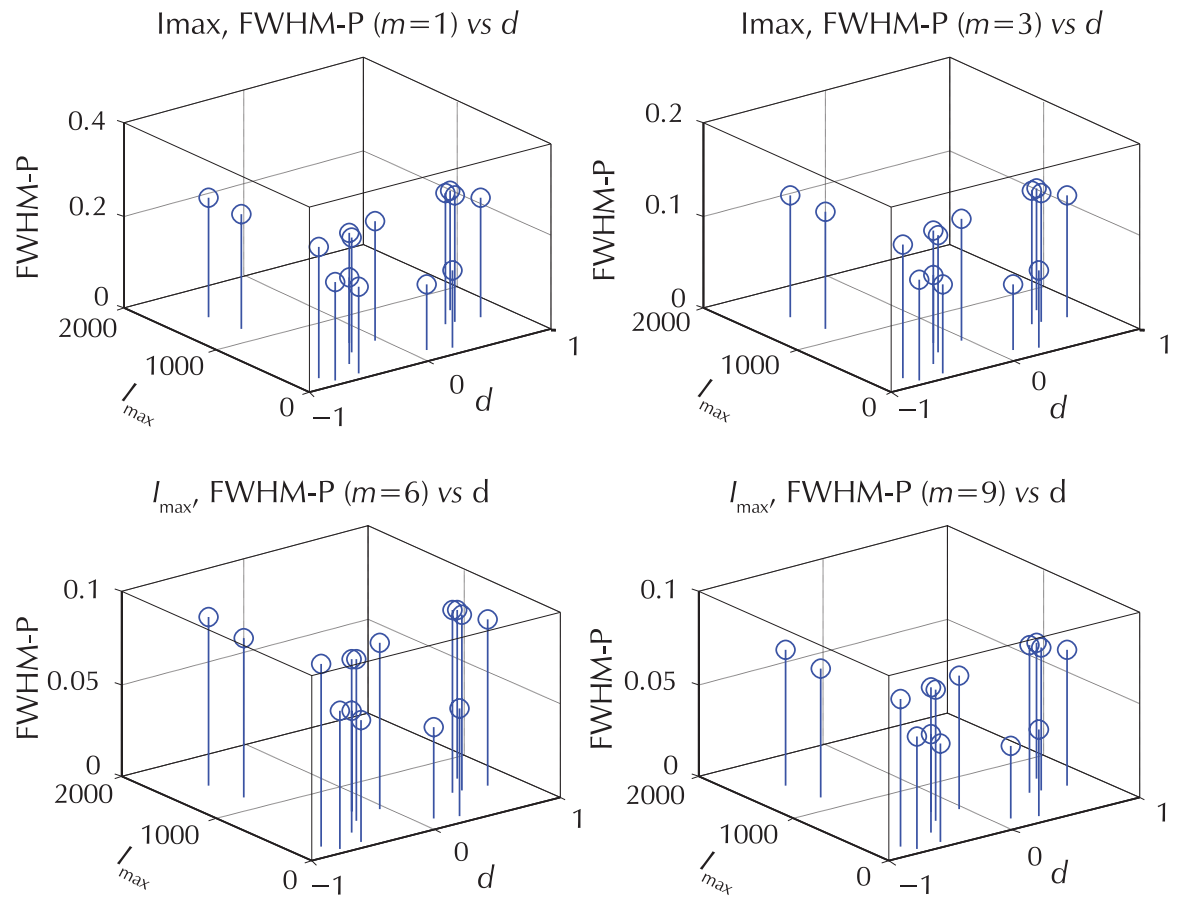

Fig. 9 - FWHM, $I_{\max }$ vs d-spacing of the XRD patterns of Pearson VII for powers of $m=1,3,6,9$
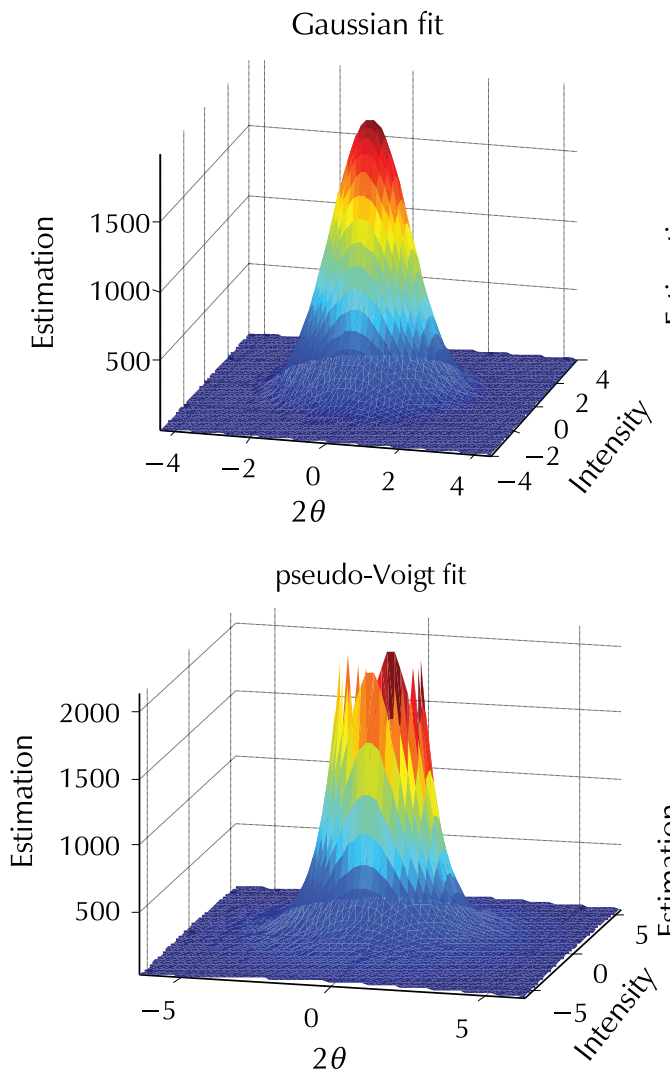

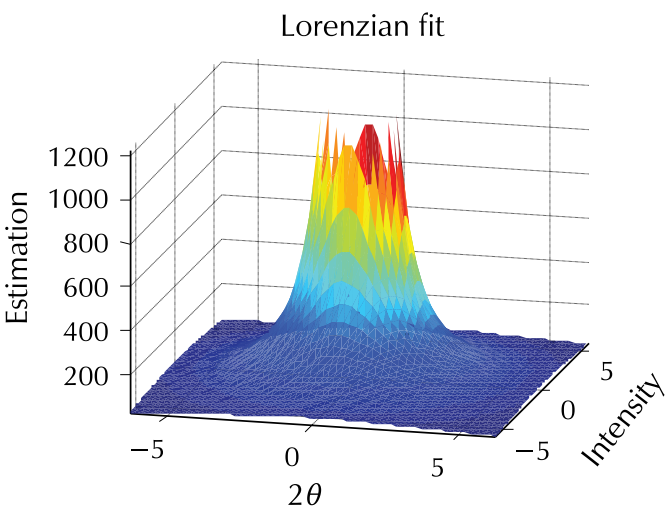

The Voigt fit

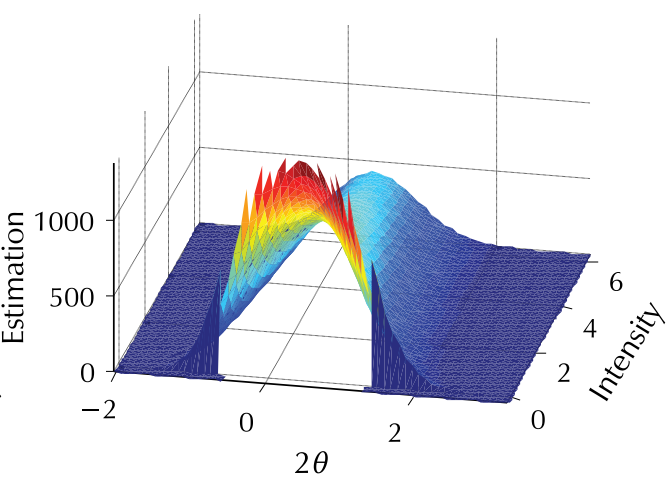

Fig. 10 - Simulations of the XRD pattern using Gaussian, Lorentzian, pseudo-Voigt, and Voigt equations 

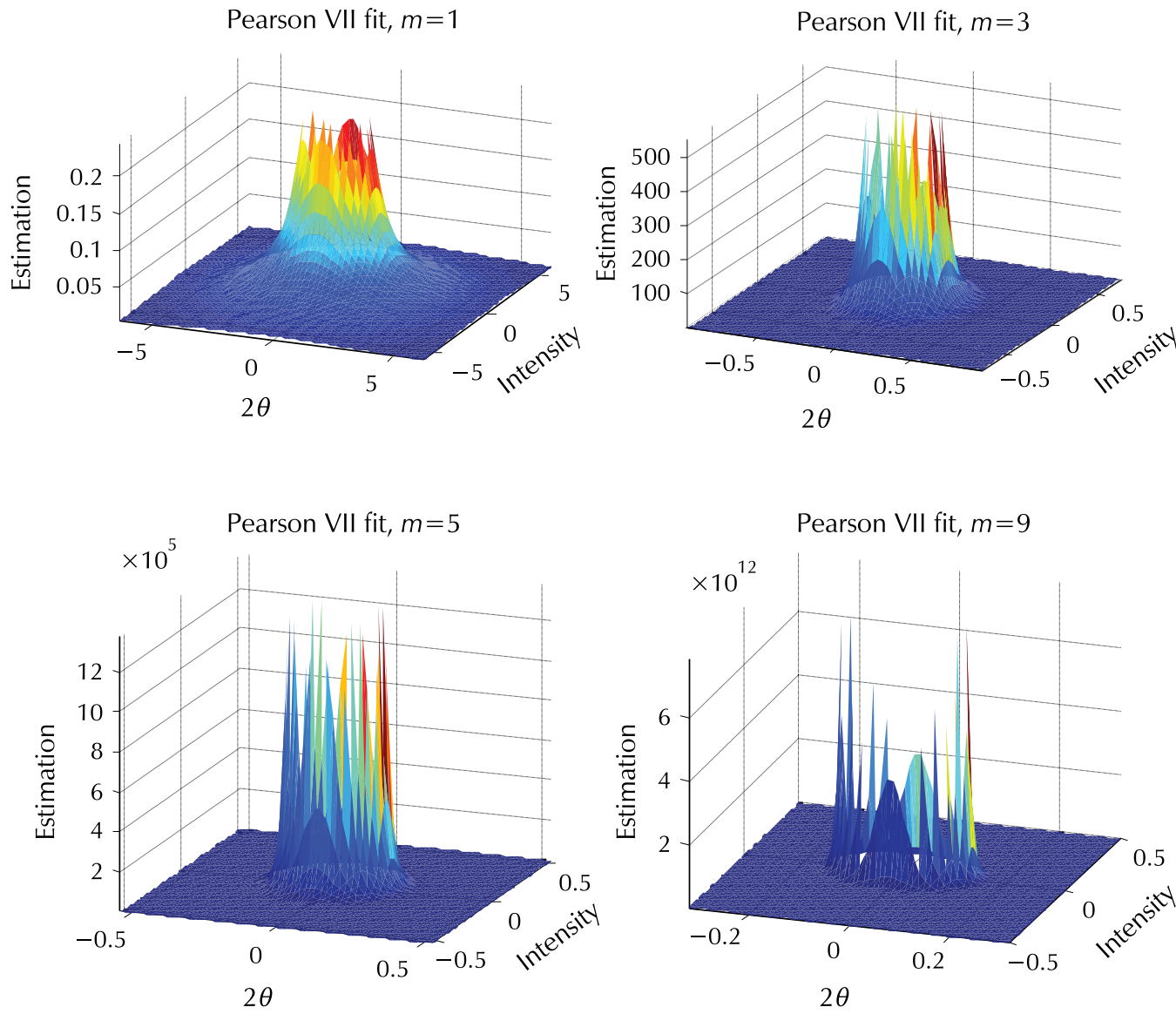

Fig. 11 - Pearson VII simulation for powers raised at $m=1,3,5,9$

The Pearson VII powers of $m=1,3,5,9$ iteration simulations of the XRD pattern were characterised as being lower sharp shaped, as seen in Fig. 11.

\section{Discussion}

A satisfactory incubation process of $A$. aceti microorganism in pyrite ash media was observed, and no toxicity occurred during the incubation, realising reasonable growth of $A$. acet in metal-sulphur mineral ash. Many subsequent validated studies on the analysis of the comprehensive diffraction pattern of the pyrite ash diffraction peaks were conducted to investigate the mineralogical composition of the metal-sulphur mineral ash. The perceived diverse XRD profile peaks of the pyrite ash sample across the profile pattern analysis are presented in Fig. 1, and the peaks correspondingly were confirmed with the previous studies of the pyrite mineral ash profiling and composition analysis figures, demonstrating comparable outcomes. ${ }^{15-16}$ Furthermore, the qualitative and quantitative analysis of the mineralogical composition of the pyrite ash samples indicated that the mineral ash mostly contained the metal-sulphur molecules of $\mathrm{FeS}, \mathrm{CuS}, \mathrm{CuSO}_{4}, \mathrm{PbS}$, and $\mathrm{PbSO}_{4}$.

Among the profiling peaks, a single peak, as illustrated in Fig. 2, was chosen for the profiling and simulation studies. The highest intensity value, -the maximum height of the chosen peak was determined as $I_{\max }=1529.17$, and the half maximum peak was found as $1 / 2 I_{\max }=764.58$, and the FWHM was observed between $\left(33.3^{\circ}\right.$ and $\left.33.54^{\circ}\right) 2 \theta$ angles at $1 / 2 I_{\max }$. The tails of the peak between $\left(33.3^{\circ}-33.54^{\circ}\right)$ $2 \theta$ angles symbolise the boundaries at left and right side of the chosen peak, attributed to the identical reflection of the lines at either side. The breadth of the peak at $0.2^{\circ}$ per step was characterised as a broad peak, indicating that the peak was symmetrical. The peak area was found to be identical to the peak intensity which exhibited the total photons observed. Error approximations sourced from the standard deviation $(\sigma)$ associated FWHM of Gaussian (FWHMG), Lorentzian (FWHML), Pearson VII (FWHMP), pseudo-Voigt (FWHMV), and Voigt (FWHMPV) functions are presented in Fig. 3 to compare the FWHM values of each profiling peak; the FWHM value of the Voigt function (FWHMV) was found to be the highest. The estimated information is presented in Figs. $4 \mathrm{a}$ and $4 \mathrm{~b}$ illustrating the prominent relations between the parameters of microstrain $(\varepsilon)$, crystallite size $(L)$, and the Bragg angle $(\theta)$, as well as between the FMHM, crystallite size (L), and Bragg angle $(\theta)$. The succeeding quoted works were done for the application of the Williamson-Hall method by plotting $\beta_{\mathrm{tot}} \mathrm{COS} \theta$ vs $\sin \theta$ to estimate the parameters of $C \varepsilon$ and $K \lambda / L$ values using the plot of $\beta$ vs $\sin \theta$, as demonstrated in Fig. 5. The strain from the slope and the size components from the intercept were attained, and the calculations of the coefficients of the parameters resulted as $C \varepsilon=0.125$ and as $K \lambda / L=-0.1$, which were used to determine the sum of 
crystallite size and strain broadening, $\beta_{\text {tot }}$. Analogous results were found between the determined average crystallite size and the particle size analysis of pyrite ash.

The ensuing pattern was obtained from strain broadening of $\beta_{\text {tot, }}$ and FWHM, $I_{\max }$ vs Bragg angle $(\theta)$ graph, as displayed in Fig. 6, to assess the relationship between the components.

The comparative presentation of microstrain parameters $\left(\varepsilon\left(\beta_{\mathrm{i}} \cos \theta\right)\right)$, integral breadth and $\cos \theta$ are presented in Fig. 7. The compared values of the simulation functions of the XRD patterns were related to half of the integral breadth $\left(\beta_{\mathrm{i}}\right)$ and adapted to the Gaussian and Lorentzian functions and their modified forms. Furthermore, the estimated FWHM data extracted from the simulation function related equations were used to simulate the FWHM, I $\max$ vs d-spacing graph, as presented in Fig. 8, which yielded the identical vision, designed to fit the observed original pattern graph from the XRD analysis. The adapted FWHM functions as FWHMG, FWHML, FWHMPV, FWHMV, and FWHMP, along with their improved graphs of FWHM, $I_{\max }$ Vs $d$-spacing from the XRD patterns for powers of $m=1,3,6,9$ were successfully projected, as shown in Figs. 8 and 9 .

While the Gaussian and Lorentzian equations were acknowledged as the basic operational peak-fitting functions, the modified Pearson VII, pseudo-Voigt, and Voigt functions were conceivably enhanced using the peak profiling parameters. The simulations, as illustrated in Figs. 10 and 11, were successfully visualised in relation to the shape function parameters such as the peak heights and breadths. An ideal symmetrical round-top fit of Gaussian simulation of the XRD pattern is illustrated in Fig. 10, and the other Lorentzian, pseudo-Voigt, and Voigt functions resulted in sharp rather than rounded tops and incomplete demonstrations. Moreover, the maximum height of the peak of the Gaussian simulation was determined as the highest intensity value, which is considered to be a similar value than the experimental observation. The more sophisticated and convoluted Voigt equation related with the Faddeyeva complex error function yielded ineffective and unproductive simulation replication in the pattern profiling. The maximum height of the peak of the Gaussian simulation was determined as highest intensity value, $I_{\max }=1529.17$ which was considered to be equal in value to the experimental observation. The Gaussian function with a round-top peak was deemed to be the most suitable profile simulation by offering a comparable maximum height of the peak $I_{\max }$ and tails at the left and right side of the chosen peak.

The Voigt equation related to the Faddeyeva complex error function was visualised ineffective and unproductive simulation replication in the pattern profiling. Fig. 11 illustrates a notable declining envision among the simulation graphs of Pearson VII of the XRD pattern with powers of $m=1,3,6,9$ iteration and simulation with powers of $m=3$, which gave the closer intensity estimation to the observed value. The Pearson VII power simulations were characterised as having a sharp-shaped appealing and smoothly decreasing fit, and the contributions from the increasing $m$ power values were yielded to obtain decreasing shape sizes for the evaluated peak.

\section{Conclusion}

No toxic effect was observed on the growth of $A$. aceti in the pyrite ash mineral pulp media, and the $\mathrm{pH}$ was reduced from 6.7 to 3.4 during the incubation. The fundamental functions were successfully applied for profiling and simulation of the chosen peak profile of pyrite ash incubated in $A$. aceti containing media. Additionally, the effectual assessment of the layer spacing, crystal size, and strain broadening, and a qualified simulation of the separate peak of the XRD pattern were achieved, and the profile shapes were interrelated with the previous pyrite mineral ash profiling studies. The determined FWHM values from the XRD profile shapes in relation to half of the integral breadth $(\beta)$ were adapted to the simulation functions of the Gaussian and Lorentzian equations and their modified forms. Moreover, FWHMG, FWHML, FWHMP, FWHMPV, FWHMV represent FWHM approximations of Gaussian, Lorentzian, pseudo-Voigt, and Voigt functions, respectively, and Pearson VII FWHM, vs $d$-spacing of the XRD patterns for powers at $m=1,3,6,9$. The functions were used for the determination of the relative values of the FWHM. As the lines almost asymptotically approached the highest intensity, the maximum height of the peak was recorded as $I_{\max }=1529.17$. The FWHM of the peak was detected as a horizontal line size at half maximum peak height between $\left(33.3^{\circ}-33.54^{\circ}\right) 2 \theta$ angles at $1 / 2 I_{\max }$. The errors in line broadening were correlated with FWHM equations of the generalised profiling functions and associated with the standard deviation $(\sigma)$ correlated FWHM equations of the profiling functions. The heights and breadths of the peak projected from the 3D simulations practically showed righteous conception with Gaussian function indicating better fit and $\sigma$ values rather than the other profiling functions which need to be refined. The Gaussian function with a round top peak was determined the most suitable profile simulation by offering a maximum height of the peak, and the left and right tails comparable to those in the experimental peak. However, the simulation by Voigt convolution resulted in an incomplete representation, possibly due to the application of the error function. Also, the reproduced peak shapes by Pearson VII raised by different $m=1,3,5,9$ power values, offered the most deficient simulations. 


\section{References \\ Literatura}

1. J. I. Langford, A. J. C. Wilson, Scherrer after sixty years: A survey and some new results in the determination of crystallite size, J. Appl. Cryst. 11 (1978) 102-113, doi: https://doi. org/10.1107/S0021889878012844.

2. V. Elser, The diffraction pattern of projected structures, Acta Cryst. A42 (1986) 36-43, doi: https://doi.org/10.1107/ S0108767386099932

3. R. J. Wells, J. Quant. Spectrosc. Ra. 62 (1999) 29-48, doi: https://doi.org/10.1016/S0022-4073(97)00231-8.

4. D. Balzar, X-Ray Diffraction line broadening: Modeling and applications to High- $T_{\mathrm{c}}$ superconductors, J. Res. Natl. Inst. Stand. Technol. 98 (3) (1993) 321-353, doi: https://doi. org/10.6028/jres.098.026.

5. G. Balakrishnan, Z. Abdul, S. Jungll, Study of $\mathrm{Al}_{2} \mathrm{O}_{3} /$ $\mathrm{ZrO}_{2}(5 \mathrm{~nm} / 20 \mathrm{~nm})$ nanolaminate composite, Composite Res. 26 (2012) 60-65, doi: https://doi.org/10.7234/ kscm.2013.26.1.60.

6. G. K. Williamson, W. H. Hall, X-ray line broadening from filed aluminium and wolfram, Acta Metall. 1 (1953) 22-31, doi: https://doi.org/10.1016/0001-6160(53)90006-6.

7. A. K. Zak, W. H. A. Majid, M. E. Abrishami, R. Yousefi, X-ray analysis of ZnO nanoparticles by Williamson-Hall and size-strain plot methods, Solid State Sci. 13(1) (2011) 251-256, doi: https://doi.org/10.1016/j.solidstatesciences.2010.11.024.

8. G. K. Wertheim, M. A. Butler, K. W. West, D. N. Buchanan, Rapid approximation to the Voigt/Faddeeva function and its derivatives, Rev. Sci. Instr. 45 (11) (1974) 1369-1371, doi: https://doi.org/10.1063/1.1686503.

9. A. K. Hui, B. H. Armstrong, A. A. Wray, Rapid computa- tion of the Voigt and complex error functions, J. Quant. Spectrosc. Ra. 19 (5) (1978) 509-516, doi: https://doi. org/10.1016/0022-4073(78)90019-5.

10. J. A. C. Weideman, Computation of the complex error function, SIAM J. Numer. Anal. 31 (5) (1994)1497-1518, doi: https://doi.org/10.1137/0731077.

11. S. M. Abrarov, B. M. Quine, Efficient algorithmic implementation of the Voigt/complex error function based on exponential series approximation, Appl. Math. Comput. 218 (5) (2011) 1894-1902, doi: https://doi.org/10.1016/j. amc.2011.06.072.

12. T. Ida, M. Ando, H. Toraya, Extended pseudo-Voigt function for approximating the Voigt profile, J. Appl. Cryst. 33 (6) (2000) 1311-1316, doi: https://doi.org/10.1107/ s0021889800010219.

13. J. I. Langford, D. Louër, E. J. Sonneveld, J. W. Visser, Applications of total pattern fitting to a study of crystallite size and strain in zinc oxide powder, Powder Diffr. 1 (3) (1986) 211221, doi: https://doi.org/10.1017/S0885715600011738.

14. Y. Liu, J. Lin, G. Huang, Y. Guo, C. Duan, Simple empirical analytical approximation to the Voigt profile, J. Opt. Soc. Am. B. 18 (2001) 666-672, doi: https://doi.org/10.1364/ JOSAB.18.000666.

15. M. L. S. Oliveira, C. R. Ward, M. Izquierdo, C. H. Sampaio, I. A. S. de Brum, R. M. Kautzmann, Chemical composition and minerals in pyrite ash of an abandoned sulphuric acid production plant, Sci. Total Environ. 430 (2012) 34-47, doi: https://doi.org/10.1016/j.scitotenv.2012.04.046.

16. N. Tugrul, E. M. Derun, M. B. Pişkin, A. Ekerim, A study on the structural behavior of reduced pyrite ash pellets by XRD and XRF analysis, Waste Manag. Res. 27 (2009) 281-287, doi: https://doi.org/10.1177/0734242X08090404. 


\title{
SAŽETAK
}

Simulacijsko modeliranje rendgenske difrakcije uzoraka piritnog pepela bioprocesiranog u medijima koji sadrže Acetobacter aceti

\author{
Yakup Ermurat
}

Fenomenalne funkcije profiliranja primijenjene su za simulaciju rendgenske difrakcije (XRD) uzorka piritnog pepela bioprocesiranog u medijima koji sadrže Acetobacter aceti. Vrijednosti širine linije na polovici visine (FWHM) procijenjene su za svaki uzorak piritnog pepela. Braggova jednadžba primijenjena je za određivanje razmaka atomskog sloja, dok su za veličinu kristalita i širenje deformacija primijenjene Scherrerove i Williamson-Hallove jednadžbe. Pogreške u proširivanju linija povezane su s jednadžbama generaliziranih funkcija profiliranja FWHM, a mikronaprezanje $(\varepsilon)$ je procijenjeno odnosom između integralne širine $\left(\beta_{\mathrm{i}}\right)$ i $\cos \theta$. Nadalje, XRD uzorak diskretnog vrha prikazan je 3D simulacijom primjenom Gaussove, Lorentzianove, Pearsonove VII, pseudo-Voigtove i Voigtove funkcije. Gaussova funkcija s okruglim vrhom pokazala se najprikladnijom simulacijom profila nudeći maksimalnu visinu vrha, $I_{\max }$ i krajeve usporedive s eksperimentalnim vrhom.

\section{Ključne riječi}

Rendgenska difrakcija uzorka, veličina kristalita i širenje deformacija, ispitivanje FWHM-a, modeliranje profila, simulacija uzorka, piritni pepeo, Acetobacter aceti

Bolu Abant Izzet Baysal University Department of Chemical Engineering Bolu, Turska
Izvorni znanstveni rad Prispjelo 5. svibnja 2020. Prihvaćeno 10. rujna 2020. 